not correlated well with AVS $(r=0.34)$.

All of the patients felt better when they left the emergency room with an AVS from 20 to 30 points lower. The intervention time was 45 minutes for traumatic patients, and 81 minutes for patients with abdominal pain and visceral surgery. The effectiveness time on pain is 86 minutes on the average after admission: it has absolutely nothing to due with the initial pain intensity. It is linked directly with the initiating time of treatment.

Discussion: It is difficult to evaluate the levels of pain in an emergency room because: (1) it relies on different kinds of pains and pathologies, (2) treatment needs to be initiated quickly, and (3) it concerns numerous kinds of medical staffs with different backgrounds. If the AVS remains the reference, the use of a simple verbal scale is enough in an emergency room. This immediate evaluation should allow a quick initiation of analgesic treatments (analgesic drugs, physical means) with a short action time.

The current effort will concentrate on the simple, but immediate, evaluation of the pain, and on the analgesic treatment (pharmacological as well as physical).

Key words: analgesia; evaluation; nurses; pain; patients; physicians; treatment

Prehosp Disast Med 2001;16(2):s34.

\section{Principles of a Multilevel Preparation System for an Extreme Situation}

F. Gusev; Ivochkin; I.V. Plushch; S.V.Beloglasova

Novosibirsk Regional Center for Disaster Medicine, Novosibirsk, RUSSIA

Disaster Medicine (DM) is a constituent of a state system emergency reaction in extreme situations (ES). In a complete approach to a disaster, DM is invoked to take preventive measures following the occurrence of a hazard that produces massive sanitary losses and for the prevention of negative medical consequences of accidents and failures. Training of the population is required for survival, with the population being responsible for rendering first medical care in ES.

For this difficult task (both for the state, and for the society), the Novosibirsk Regional Center for Disaster Medicine provided the following methodological principles for a multilevel system to prepare the population to be able to understand the psychology of conduct, the determinants of survival, and render first aid to themselves as important actions in ES.

This package approach to the training a population that may or may not have a medical education proved that the optimal approach is the combined programs of initial and a continuous process of training with gradual escalation of the information during life (up to school, the educational institutions, army, etc.). In addition, it also presents uniform criteria as algorithms of actions and also of a rating of knowledge of the population, and will facilitate the development of a continual skill set under the preset program.

The system examines the complex decision making of the tasks, and must be taken before a service, as it will be a required condition for the safety of vital activities and the shaping of personal safety.

Key words: disasters; population; safety; self-care; survival; training

Prehosp Disast Med 2001;16(2):s34.

\section{A Simulation Model of Biological Hazard or Widespread Infectious/ Biological Disaster Focusing on the Characteristics of the Spread of Disease by the Percolation-Diffusion Theory \\ Y. Haraguchi; Y. Tomoyasu; T. Ishihara; T. Nagata; \\ M. Sakai; M. Hoshino; K. Mabuchi}

Tachikawa, Tokyo, JAPAN

Objective: The aim of this study is to create a simulation model of widespread biological hazard and to review its significance.

Methods: The simulation model is created by using the percolation-diffusion theory. The contagion rate, incubation period, infectious period, diseased period, and mortality are arbitrary. In this model, it is premised that no treatment is applied.

Results and Conclusions: This study is preliminary. The results are quite complex. However, parameters that result in an increase in the number of infected patients (infinitely) are a high contagion ratio and long infectious period. However, the number of infected patients will be depressed if mortality rate is high.

Key words: hazard, bioloigical; computer; contagion rate; infection; incubation period; model; mortality;

percolation-diffusion theory

E-mail: akisatoh@mxm.mesh.ne.jp

Prebosp Disast Med 2001;16(2):s34.

\section{Stroke Patients in the Emergency Medical Services (EMS)}

F. Heid; C. Lott; H.J. Hennes

Clinic of Anesthesiology, Johannes Gutenberg-University Hospital, Mainz, GERMANY

Introduction: Stroke represents one of the major health care challenges in the world today. Early initiation of therapy can improve outcome. There is increasing interest in the role of the EMS in early stroke therapy, in view of the fact that time is one of the critical factors in stroke treatment. The aim of our study was to analyze time intervals of stroke treatment in the EMS.

Methods: First, all advanced life support units and basic ambulances of a German community EMS-system were equipped with questionnaires that were completed after prehospital treatment of patients with suspected stroke. Secondly, patients with suspected stroke were surveyed by a member of our group. The relevant time intervals and anamnestic and clinical data were documented.

Results: Data of 700 patients were obtained: $38 \%$ of patients suffered an acute ischemic stroke; in $12 \%$, symptoms were due to intracranial hemorrhage. Thirty-eight percent were treated by an emergency physician at the scene; 50\% received EMS treatment within 2 hours; and 